\title{
Star-shaped polyhedral oligomeric silsesquioxane-polycaprolactone-polyurethane as biomaterials for tissue engineering application
}

\author{
Choon Peng Teng ${ }^{1,2}$, Khine Yi Mya ${ }^{1}$, Khin Yin Win ${ }^{1}$, Chee Chuan Yeo ${ }^{1}$, Michelle Low ${ }^{1}$, Chaobin $\mathrm{He}^{1,3}$ and \\ Ming-Yong $\operatorname{Han}^{1,2}$
}

Polyhedral oligomeric silsesquioxane (POSS) is a unique molecule that is composed of an inorganic silica core with eight organic functional arms, which can be used as a nucleus for covalent bonding to create star-shaped block copolymers with improved mechanical and biological properties. In this work, highly porous star-shaped POSS-polycaprolactone-polyurethane (POSS-PCLPU) films were synthesized as scaffold biomaterials for tissue engineering. These films have an interesting morphology consisting of rough spherulites with filamentous structures spreading out from their centers; the unique nanotopography was shown to be suitable for cell growth. In vitro degradation was monitored for $\mathbf{5 2}$ weeks in terms of the weight loss and morphology changes of the films. The degradation profile exhibited a slow initial weight loss of $<1 \%$ during the first 24 weeks, followed by a rapid weight loss of $\sim 18 \%$ in the following 28 weeks. The films demonstrated excellent biocompatibility and cell-substrate affinity, with a high cell viability of $>95 \%$ and rapid cell proliferation. The high porosity, unique surface nanotopography and excellent biocompatibility of the star-shaped POSS-PCL-PU film make it a great candidate as a tissue engineering scaffold biomaterial. NPG Asia Materials (2014) 6, e142; doi:10.1038/am.2014.102; published online 7 November 2014

\section{INTRODUCTION}

Tissue engineering has emerged as a promising treatment to improve or replace the biological functions of the damaged tissue or organs. Tissue engineering involves the use of a combination of cells, engineered materials and suitable biochemical factors for the growth of donor or patient cells in a three-dimensional scaffold that acts as a structural template upon which cells can adhere, proliferate and eventually form new tissues. ${ }^{1,2}$ Ideally, scaffolds should be biocompatible to prevent inflammation or toxicity reactions in vivo, and they should also be highly porous to allow for the cellular infiltration and nutrition supply via diffusion, convection and directed fluid flow during the culture. ${ }^{3}$ It is advantageous when scaffolds have a structure similar to the extracellular matrix for cell guidance and cell-matrix interactions to facilitate neotissue development. ${ }^{4}$ It is also important to have controlled degradation rates to provide the required support for tissue formation, allowing degradation to start as neotissue forms. The products of degradation should be biocompatible to ensure that there is no toxicity. ${ }^{5}$

Block copolymers are attractive scaffold materials because their properties, such as porosity, biodegradability and mechanical strength, can be appropriately designed and specifically tuned to match the required tissue engineering application. ${ }^{6}$ Polyhedral oligomeric silsesquioxane (POSS) is composed of an inorganic silica cube formed by rigid $\mathrm{Si}-\mathrm{O}$ bonds with eight organic functional $\mathrm{arms}^{7-11}$ that are reactive with other polymers via covalent bonding to form hybrid starshaped block copolymers. ${ }^{12}$ POSS-based materials exhibit enhanced thermal and mechanical stability arising from the inorganic properties and structural features of the POSS core. In addition, these hybrid materials also offer great versatility and can be used in the development of advanced biomaterials. ${ }^{11}$ For example, POSS can be incorporated with polycaprolactone (PCL), ${ }^{13}$ polyurethane (PU), ${ }^{14}$ polycarbonate urethane urea ${ }^{15}$ and polycarbonate urethane ${ }^{16}$ for improved biological and mechanical properties to achieve better processability, increased strength, toughness and higher stability for tissue engineering applications. ${ }^{17}$ Oseni et $a l^{16}$ reported that the surface topography of POSS enabled nanoscale interactions of cells via a scaffold matrix, which increased the viability of chondrocytes on POSS-polycarbonate urethane scaffolds compared with PCL scaffolds. Knight et al $l^{18}$ reported that there was minimal weight loss for POSS-incorporated polyester PU films in the earlier stage, followed by a large mass loss at the later stage of in vitro degradation, and the hybrid films also exhibited excellent biocompatibility with no inflammatory responses. POSS-PU hybrids were reported ${ }^{19-21}$ to possess improved properties, such as higher thermal stability, higher oxidative resistance and increased mechanical strength.

In this study, we report the use of a star-shaped POSS-PCL-PU polymer as a scaffold material in tissue engineering applications. POSS was used as the starting inorganic core to introduce three-dimen-

${ }^{1}$ Institute of Materials Research and Engineering, Singapore, Singapore; ${ }^{2}$ Department of Biomedical Engineering, National University of Singapore, Singapore, Singapore and ${ }^{3}$ Department of Materials Science \& Engineering, National University of Singapore, Singapore, Singapore

Correspondence: Dr KY Win or Professor M-Y Han, Institute of Materials Research and Engineering, 3 Research Link, Singapore 117602, Singapore.

E-mail: yw-khin@imre.a-star.edu.sg or my-han@imre.a-star.edu.sg

Received 9 June 2014; revised 10 September 2014; accepted 15 September 2014 
sionality and to improve the surface nanotopography. By incorporating organic groups in the robust core through extension with PCL and cross-linking with PU, the resulting unique inorganic-organic nanocomposite can achieve various functionalities, such as enhanced cell attachment, biodegradability and stability of the overall structure. The star-shaped POSS-PCL-PU films were investigated regarding their properties, such as porosity, hydrophobicity and surface topography.
In addition, degradation studies and cytotoxicity and biocompatibility tests were performed to access the suitability of the films as tissue engineering scaffolds. The POSS-PCL-PU films exhibit a high porosity of $>75 \%$ and intrinsic nanoscale features that enhance cell attachment and growth. The films exhibited negligible weight loss during the initial 24 weeks of degradation, followed by a large increase in the weight loss of $18 \%$ in the following 28 weeks. Most importantly, the

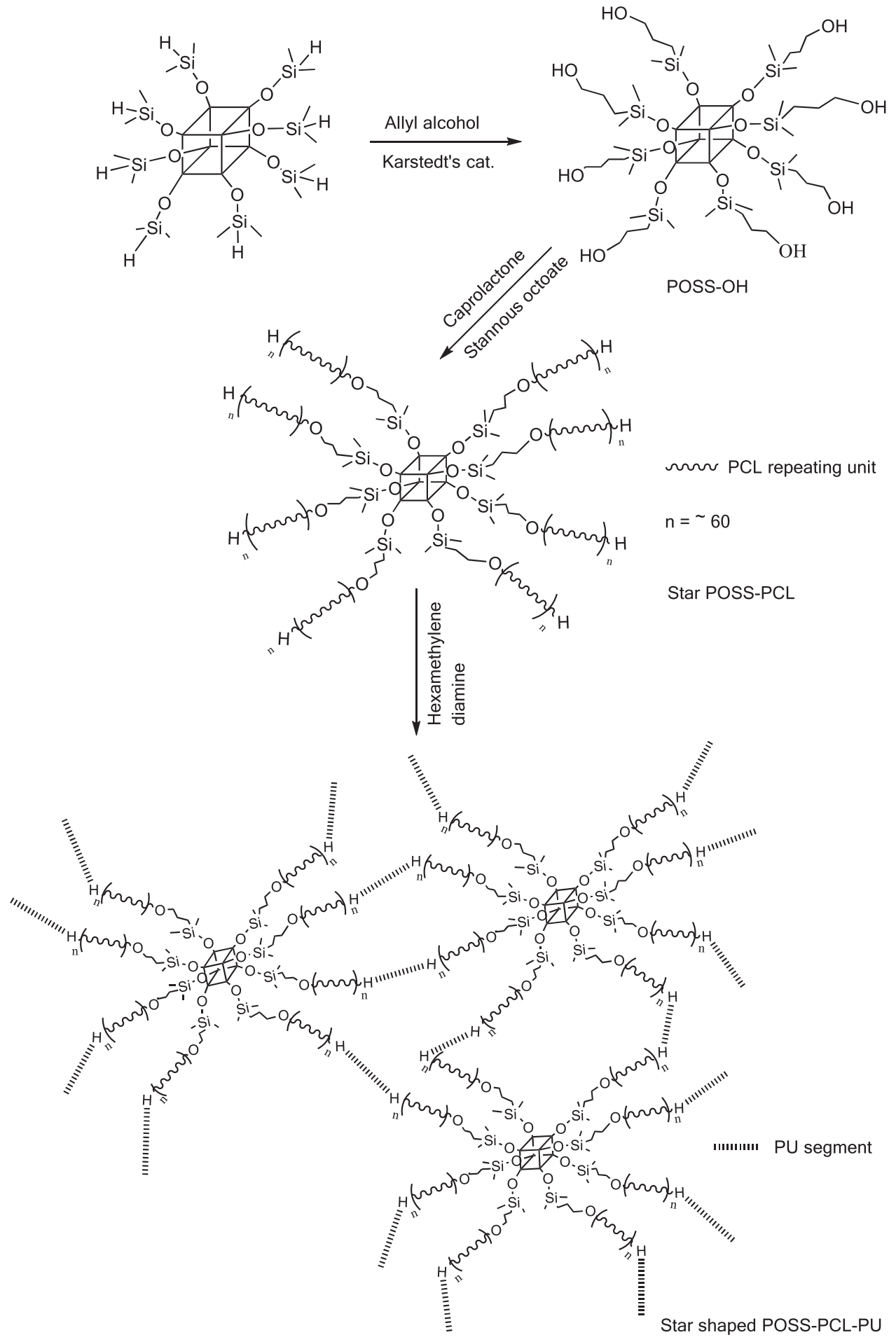

Figure 1 Schematic synthesis of star-shaped POSS-PCL-PU. 
POSS-PCL-PU films are highly biocompatible, as cell viability is still maintained at $>95 \%$, even after $48 \mathrm{~h}$ of incubation with the byproducts of degradation.

\section{MATERIALS AND METHODS}

Materials

POSS (POSS, $M_{w}=1017.99$ ) was purchased from Hybrid Plastics Inc. (Hattiesburg, MS, USA) and dried under vacuum at $60^{\circ} \mathrm{C}$ for $24 \mathrm{~h}$ prior to use. $\varepsilon$-Caprolactone was purchased from Tokyo Chemical Industry (Tokyo, Japan) and distilled over calcium hydride under reduced pressure prior to use. PCL $\left(M_{w}=80000\right)$, allyl alcohol ( $\left.\geqslant 99 \%\right)$, hexamethylene diisocyanate (99\%), stannous octoate $(95 \%)$, dibutyltin dilaurate $(95 \%)$ and fluorescein diacetate were obtained from Sigma-Aldrich (St Louis, MO, USA). alamarBlue cell viability assay was purchased from Life Technologies (Grand Island, NY, USA). Platinum divinyltetramethyldisiloxane was supplied by Aldrich and diluted to $2 \mathrm{~mm}$ solution in anhydrous toluene for use as a Karstedt's catalyst. Toluene was freshly distilled over sodium benzophenone under nitrogen prior to use. The other chemicals were used as received without further purification.

\section{Synthesis of star-shaped POSS-PCL}

Hydroxyl terminated POSS (POSS-OH) was first synthesized with the use of allyl alcohol as the monomer and platinum divinyltetramethyldisiloxane as a Karstedt's catalyst according to previous reports. ${ }^{18-21}$ The proton nuclear magnetic spectrum of POSS-OH is shown in Supplementary Figure S1a. The disappearance of the $\mathrm{Si}-\mathrm{H}$ proton resonance peak at 4.72 p.p.m in proton nuclear magnetic resonance indicated that all eight corners of the POSS core were substituted with the hydroxyl group. A batch of star-shaped POSS-PCL was then synthesized by using a 1:480 ratio of POSS-OH to $\varepsilon$-caprolactone. In a typical procedure, POSS-OH $(0.1 \mathrm{~g}, 0.07 \mathrm{mmol}), \varepsilon$-caprolactone $(3.84 \mathrm{~g}, 33.60$ $\mathrm{mmol}$ ) and stannous octoate $(3.72 \mathrm{mg}, 0.01 \mathrm{mmol})$ were mixed into a predried Schlenk flask followed by five cycles of freeze-pump-thaw degassing of the mixture under magnetic stirring. The flask was then sealed and immersed in a thermostat oil bath at $120^{\circ} \mathrm{C}$ for $12 \mathrm{~h}$. The crude product was dissolved in a
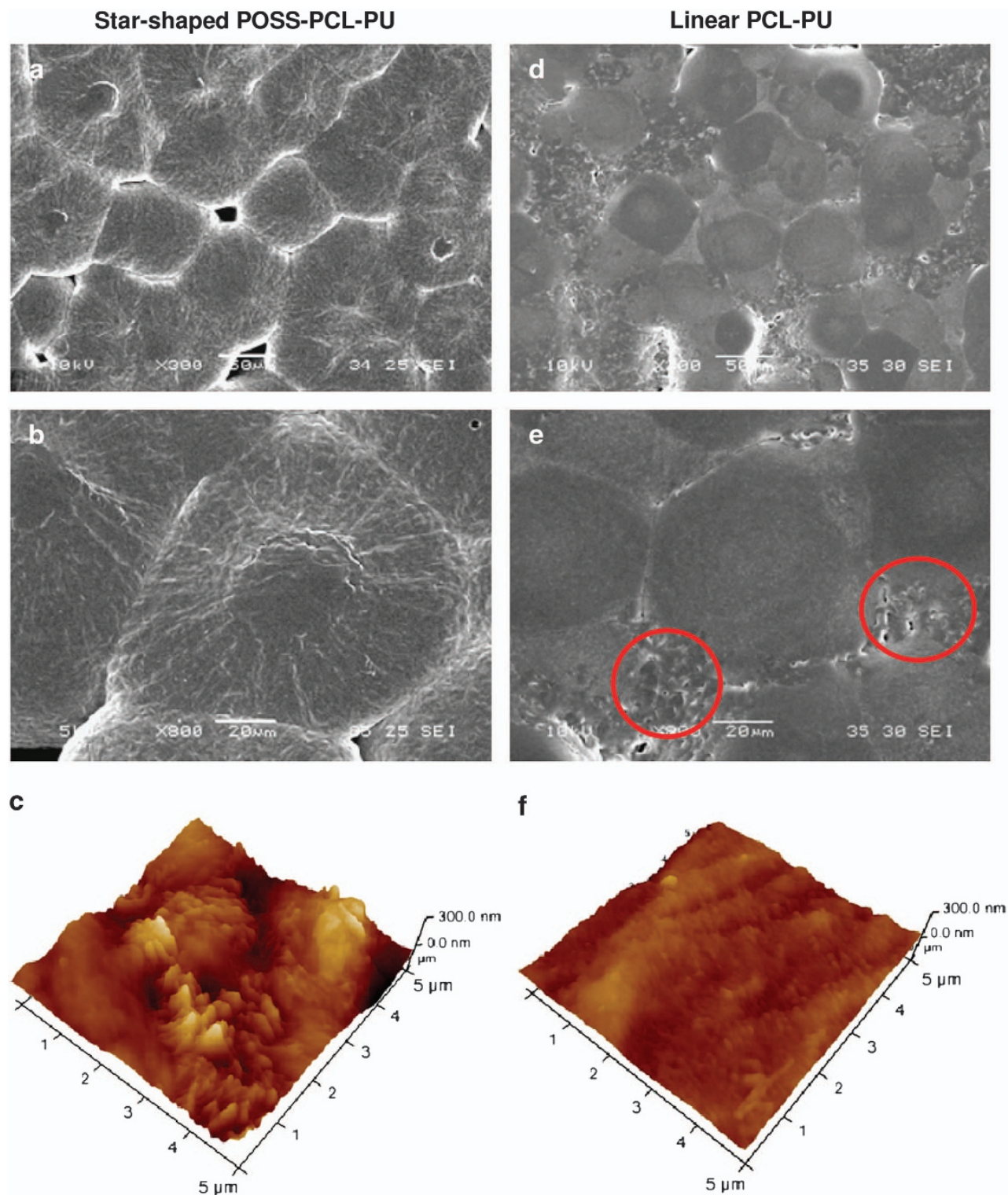

Figure 2 Morphology of star-shaped POSS-PCL-PU film observed in SEM images at (a) $300 \times$ magnification, (b) $800 \times$ magnification and (c) close-up threedimensional AFM image. Morphology of linear PCL-PU film observed in SEM images at (d) $300 \times$ magnification, (e) $800 \times$ magnification and (f) close-up three-dimensional AFM image of $5 \times 5 \mu \mathrm{m}$ scanning across the film. Scale bar $=50 \mu \mathrm{m}$ in (a) and (d); scale bar $=20 \mu \mathrm{m}$ in (b) and (e). 
minimum amount of chloroform and then precipitated in an excess amount of cold methanol. After repeating the precipitation process three times, the obtained product was dried in a vacuum oven overnight at $40{ }^{\circ} \mathrm{C}$ with an yield of $>98 \%$. The as-prepared POSS-PCL was characterized via measurement of the proton nuclear magnetic resonance spectrum, as shown in Supplementary Figure S1b. Proton nuclear magnetic spectrum $\left(\mathrm{CDCl}_{3}, 400\right.$ $\mathrm{MHz}$, p.p.m): $0.14 \quad\left(\mathrm{Si}\left(\mathrm{CH}_{3}\right)_{2} \mathrm{CH}_{2}\right) ; 0.61 \quad\left(\mathrm{Si}\left(\mathrm{CH}_{3}\right)_{2} \mathrm{CH}_{2}\right) ; 1.68 \quad\left(\mathrm{Si}\left(\mathrm{CH}_{3}\right)_{2}\right.$ $\left.\mathrm{CH}_{2} \mathrm{CH}_{2} \mathrm{CH}_{2}-\mathrm{PCL}\right) ; 4.06\left(\mathrm{O}-\mathrm{CO}-\left(\left(\mathrm{CH}_{2}\right)_{4}-\mathrm{CH}_{2}-\right)_{\mathrm{n}}\right) ; 3.65\left(\mathrm{O}-\mathrm{CO}-\left(\mathrm{CH}_{2}\right)_{4}-\right.$ $\left.\mathrm{CH}_{2}-\mathrm{OH}\right) ; 2.26-2.30\left(\mathrm{O}-\mathrm{CO}-\mathrm{CH}_{2}-\left(\mathrm{CH}_{2}\right)_{4}\right) ; 1.60-1.68 \quad\left(\mathrm{O}-\mathrm{CO}-\mathrm{CH}_{2} \mathrm{CH}_{2}-\right.$ $\left.\left(\mathrm{CH}_{2}\right)_{3}\right)$; and 1.34-1.41 (O-CO- $\left.\mathrm{CH}_{2} \mathrm{CH}_{2} \mathrm{CH}_{2} \mathrm{CH}_{2} \mathrm{CH}_{2}\right)$. In our star-shaped POSS-PCL polymer, the integral ratio of $-\mathrm{Si}\left(\mathrm{CH}_{3}\right)_{2}$ to $-\mathrm{Si}\left(\mathrm{CH}_{3}\right)_{2} \mathrm{CH}_{2}$ is 5.9:2.0, which is close to the theoretical ratio of 6:2. There is a higher proportion of $-\mathrm{CH}_{2}-\mathrm{OH}$ compared with $-\mathrm{Si}\left(\mathrm{CH}_{3}\right)_{2} \mathrm{CH}_{2}$, with an integral ratio of 2.8:2.0, compared with the theoretical ratio of 2:2, suggesting the presence of unattached sole PCL chains in the star-shaped POSS-PCL. Nevertheless, the majority of the PCL chains were attached to the POSS core to achieve a starshaped polymer, as shown by the sharp melting transition in differential scanning calorimetry thermogram, ${ }^{12}$ which is ascribed to the formation of a homogeneously cross-linked structure from the POSS core containing uniformly distributed polymer chains.

\section{Preparation of star-shaped POSS-PCL-PU films and linear PCL-PU films}

To synthesize star-shaped POSS-PCL-PU films, a stoichiometric amount of hexamethylene diisocyanate was added into a flask containing $5 \mathrm{~g}$ of as-purified POSS-PCL dissolved in $25 \mathrm{ml}$ of freshly dried chloroform (the end group, $-\mathrm{OH}$ of POSS-PCL reacted with hexamethylene diisocyanate to form the -NHCO functional group of urethane). The mixed solution was stirred to react for $6 \mathrm{~h}$ at room temperature after adding dibutyltin dilaurate as the catalyst. The solution was then poured into a polytetrafluoroethylene mold, followed by placing the mold containing the solution into a nitrogen-filled oven at room temperature for $24 \mathrm{~h}$ prior to curing at $80^{\circ} \mathrm{C}$ for $12 \mathrm{~h}$. The resulting film was washed with chloroform to remove the materials that were not cross-linked, and then the washed film was dried in a vacuum oven at $80^{\circ} \mathrm{C}$ to obtain star-shaped POSSPCL-PU films. The samples were then cut into small square pieces with dimensions of $10 \mathrm{~mm} \times 10 \mathrm{~mm} \times 1 \mathrm{~mm}$. As a comparative study, linear PCLPU was also prepared by mixing PCL and hexamethylene diisocyanate at a stoichiometric ratio and stirring for $6 \mathrm{~h}$ at room temperature, followed by performing the same curing and washing process described above. The linear PCL-PU films were also cut into square pieces of the same dimensions as POSS-PCL-PU films.

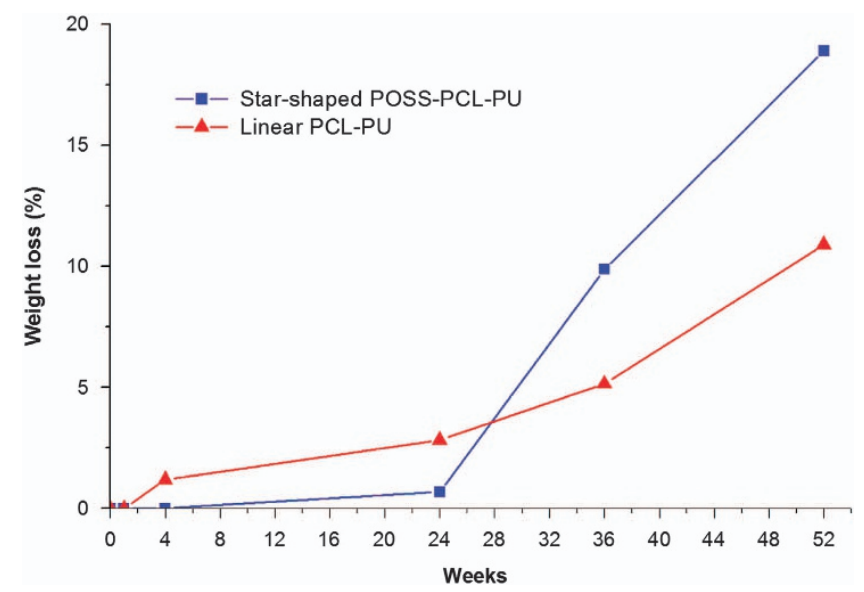

Figure 3 Weight loss percentage of star-shaped POSS-PCL-PU and linear PCL-PU films over 52 weeks. Star-shaped POSS-PCL-PU film has an extremely slow initial rate of degradation which is attributed to the POSS content. Weight loss was negligible for the first 24 weeks. There was a substantial weight loss of about $9 \%$ from week 24 to week 36 and a further $9 \%$ weight loss from week 36 to week 52 . PCL-PU film has a gradual and more consistent weight loss profile over 52 weeks.

\section{Characterization}

A JEOL 5600 scanning electron microscope (SEM) (JEOL, Tokyo, Japan) was used to characterize the surface morphology of the films. To further analyze the surface, atomic force microscopy (AFM) was performed using a Dimension ICON AFM (Bruker, Santa Barbara, CA, USA) operated in tapping mode at a scan frequency of $0.5 \mathrm{~Hz}$, using standard point probes (Nanoworld, Neuchâtel, Switzerland). Hydrophobicity of the films was determined by measuring the contact angle using NRL C.A Goniometer, Model No.100-00 from Ramé-hart (Succasunna, NJ, USA). A drop of water was dropped onto the surface of the films, and contact angle was measured from both edges of the droplet. The average contact angle was determined from 10 readings.

\section{Measurement of film porosity}

The liquid displacement method was chosen to determine the porosity of the polymer films. ${ }^{22,23}$ The criterion for the displacement liquid is that it should prevent shrinkage or swelling of the material, so that there is no change in the volume of material. In addition, the material should not dissolve in the displacement liquid. Distilled water was selected as the displacement liquid because it can easily enter the pores of the polymer sample and it does not cause changes in the size of the material. A measuring cylinder with $20 \mathrm{ml}$ of the displacement liquid was weighed and denoted as $M_{1}$. A piece of film of known weight $\left(M_{s}\right)$ was transferred into the cylinder and sonicated (Elma Transsonic Digitals Ultrasonic bath (Hilsonic, Birkenhead, UK), 100\% power) for $20 \mathrm{~min}$ to facilitate permeation of the liquid into the pores. After standing overnight, the liquid was topped up to the original level of $20 \mathrm{ml}$ and weighed $\left(M_{2}\right)$. Subsequently, the polymer film was removed, and then the cylinder was weighed $\left(M_{3}\right)$. The porosity was calculated using $\left(M_{2}-M_{3}-M_{\mathrm{s}}\right) /\left(M_{1}-M_{3}\right)$.

Weight loss and morphology changes during in vitro degradation The initial weights $\left(M_{i}\right)$ of star-shaped POSS-PCL-PU and linear PCL-PU films with dimensions of $10 \mathrm{~mm} \times 10 \mathrm{~mm} \times 1 \mathrm{~mm}$ were measured. Each individual piece was placed into a vial containing $5 \mathrm{ml}$ of phosphate buffered saline (PBS) buffer. The bottles were subsequently transferred into a water bath (Grant OLS200), with continuous shaking at 100 r.p.m at a temperature of $37^{\circ} \mathrm{C}$ for 52 weeks. The samples were taken out at predetermined time points and rinsed with distilled water and then freeze-dried for $24 \mathrm{~h}$ before measuring their final weights $\left(M_{f}\right)$. The percentage weight loss was calculated using $\left(M_{i}-M_{f}\right) /$ $M_{i} \times 100 \%$. The samples were sputter coated with gold (JEOL JFC-1200 fine coater) (JEOL) for $20 \mathrm{~s}$ and then viewed using SEM to observe the morphology changes due to degradation.

\section{Cell viability}

Five pieces of POSS-PCL-PU or PCL-PU films with a total weight of $\sim 24 \mathrm{mg}$ were incubated in $2 \mathrm{ml}$ of PBS buffer at $37^{\circ} \mathrm{C}$ for 8 weeks under shaking at 100 r.p.m. After removing the films, a solution at a concentration of 12.0 $\mathrm{mg} \mathrm{ml}^{-1}$ was obtained and then further diluted into different concentrations $\left(6.0,3.0,1.5\right.$ and $\left.0.75 \mathrm{mg} \mathrm{ml}^{-1}\right)$. Fibroblast cells (CCD-112 CoN) were seeded at a density of $2.0 \times 10^{4} \mathrm{cells} \mathrm{cm}^{-2}$ in 96-well plates (Corning) and then incubated with the above solutions for $48 \mathrm{~h}$ at $37^{\circ} \mathrm{C}$ in a humidified atmosphere containing $5 \% \mathrm{CO}_{2}$. After incubation, the viability of the cells was evaluated via alamarBlue (Life Technologies) reagent. The reducing environment of the viable cells converts the blue resazurin contents of alamarBlue (Life Technologies) reagent into highly fluorescent red resorufin, which can be measured using $570 \mathrm{~nm}$ excitation by a Tecan microplate reader. An average of five readings was taken for each concentration. The viability is calculated by the fluorescence emission values at $610 \mathrm{~nm}$ of the treated samples divided by that of the control cells without treatment and presented as a percentage viability of the control cells.

\section{Confocal imaging}

The films were washed with ethanol and double-distilled water three times before use in the cell culture. The fibroblast cells were first seeded on the POSSPCL-PU and PCL-PU films and further incubated at $37^{\circ} \mathrm{C}$ for 10 days. The culture medium was changed every alternate day. At the end of the incubation period, the cells were stained with $1 \mu \mathrm{g} \mathrm{ml}^{-1}$ of fluorescein diacetate for $20 \mathrm{~min}$ and then rinsed with PBS. Cell adhesion and proliferation were determined 

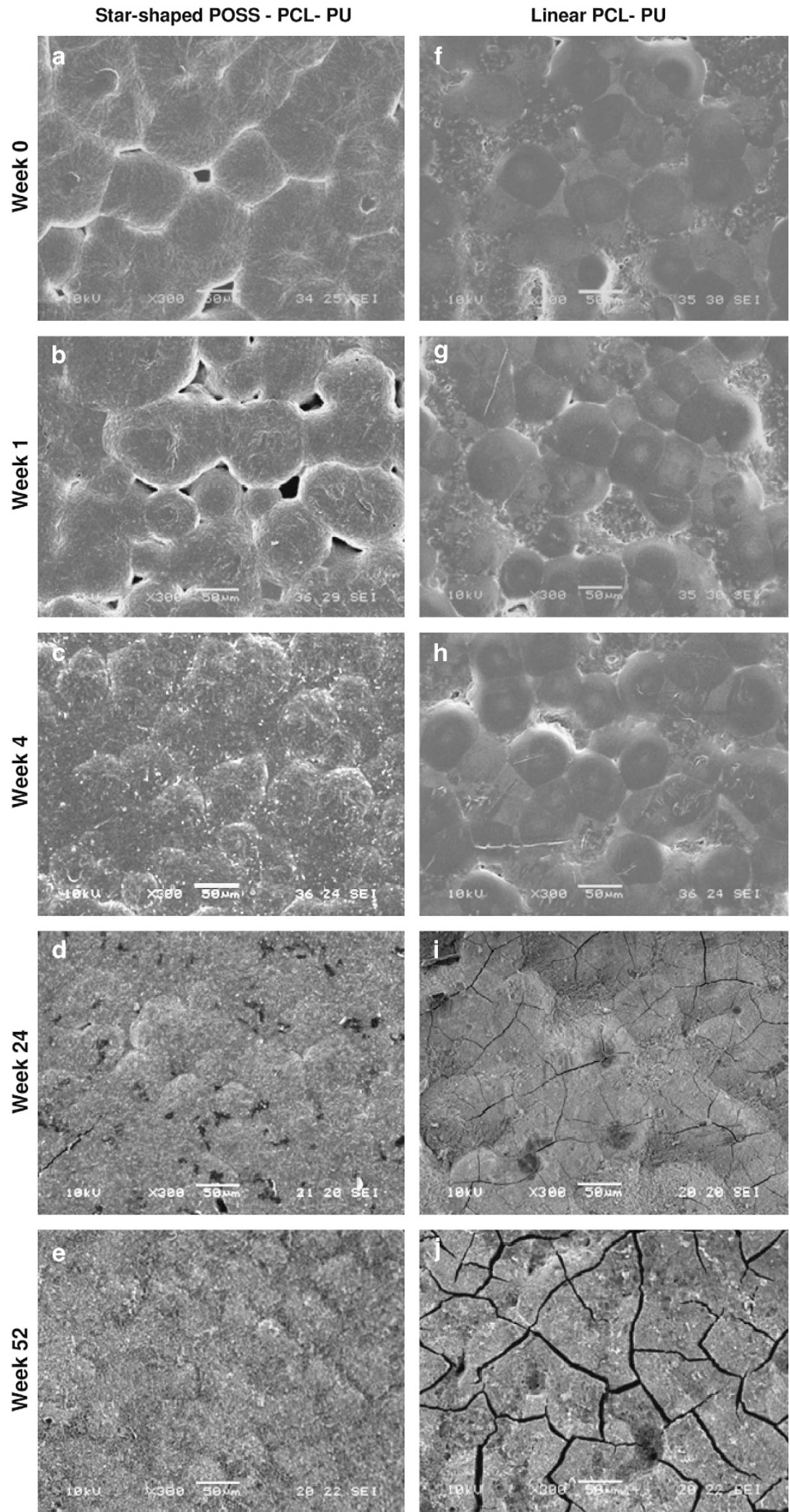

Figure 4 SEM images of star-shaped POSS-PCL-PU and linear PCL-PU films undergoing degradation for 52 weeks in PBS buffer at $37^{\circ} \mathrm{C}$. Scale bar $=50 \mu \mathrm{m}$. 

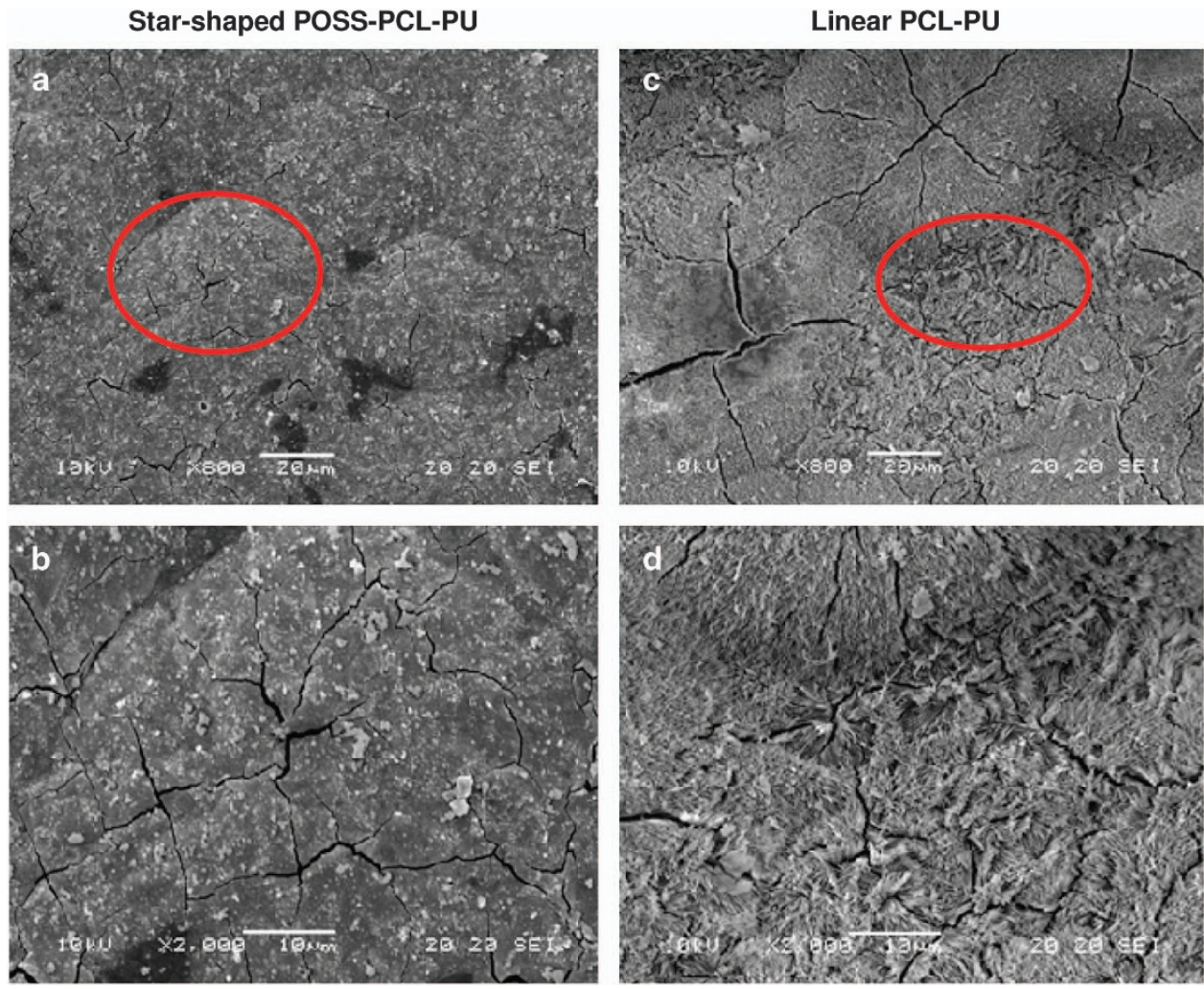

Figure 5 SEM images of star-shaped POSS-PCL-PU film at the end of 24 weeks at (a) $800 \times$ magnification and (b) $2000 \times$ magnification of the red circled area of interest in (a). SEM images of linear PCL-PU film at the end of 24 weeks at (c) $800 \times$ magnification and (d) $2000 \times$ magnification of the red circled area of interest in (c). Scale bar $=20 \mu \mathrm{m}$ in (a) and (c) scale bar $=10 \mu \mathrm{m}$ in $\mathbf{b}$ and $\mathbf{d}$.

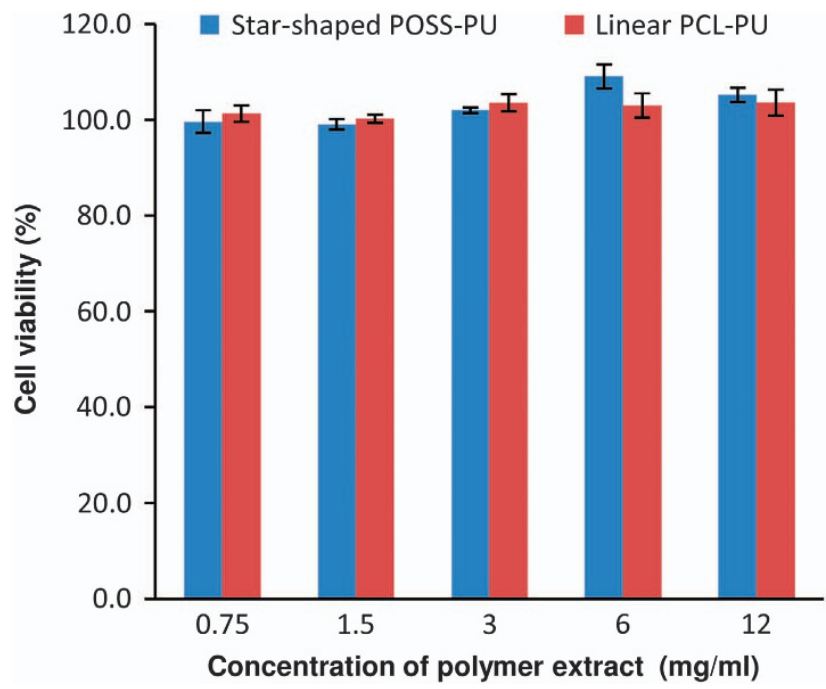

Figure 6 Cytotoxicity of the degraded products of star-shaped POSS-PCL-PU and linear PCL-PU films to fibroblast cells (CCD-112 CoN). Error bar shows standard deviation of five readings.

using a confocal microscope (FV1000 system, Olympus, Tokyo, Japan) equipped with a $20 \times$ dry objective. Z-stack images of $1024 \times 1024$ pixels were acquired with $1.5-\mu \mathrm{m}$ incremental steps over $0.0-15.0 \mu \mathrm{m}$ in the $z$ direction and processed using Olympus Fluoview (Olympus) and Imaris from Bitplane (Zurich, Switzerland). Imaris is an imaging software that allows for threedimensional rendering and cross-sectional viewing of confocal images.

\section{RESULTS AND DISCUSSION}

POSS-PCL was synthesized by reacting POSS-OH with $\varepsilon$-caprolactone extension, followed by a reaction with hexamethylene diisocyanate to form the star-shaped POSS-PCL-PU (Figure 1). By incorporating POSS in PCL-PU, which is a commonly used material in tissue engineering, the functionalities can be tailored to achieve the desired properties. To compare the differences in characteristics because of the incorporation of POSS in this nanocomposite, PCL-PU was selected as a control. Both star-shaped POSS-PCL-PU and linear PCL-PU films were characterized to determine their morphology, porosity and hydrophobicity. These films were also evaluated regarding their biodegradability, growth of cells and cytotoxicity of their degradation products. It was observed via SEM that star-shaped POSS-PCL-PU films presented tightly packed spherulites of $50-70 \mu \mathrm{m}$ in size with some gaps in between (Figures $2 \mathrm{a}$ and $\mathrm{b}$ ). These spherulites were rough with filamentous networks branching outwards in the radial direction from the center, revealing the three-dimensional structures introduced by the POSS core. The SEM analysis also revealed that the film morphology was homogenous, affirming that it is composed of mainly of POSS-PCL-PU. There was no noticeable amount of PCL-PU formed from the unattached PCL, which is postulated to be present in the mixture of POSS-PCL. In comparison, the linear PCL-PU films featured tightly packed spherulites of $40-50 \mu \mathrm{m}$ in size (Figures $2 \mathrm{~d}$ and e), which were two-dimensional and smooth. The regions around the peripheries of spherulites were porous, as highlighted by the red circles in Figure 2e. It was also observed via AFM that the POSS-PCL-PU films had a rough surface with a large number of nanosize peaks (Figure 2c), but the PCL-PU films did not exhibit these nanofeatures 
(Figure 2f). The unique morphology and nanorough surface of the star POSS-PCL-PU films observed via SEM and AFM can be attributed to the three-dimensional structure of the POSS core and the synergy of the hydrophobic moieties of POSS in combination with the PCL-PU morphology. The rough surface features of the POSS-PCL-PU films have favorable effects on cell response. ${ }^{16,24}$

The porosity of the two types of films was characterized using the liquid displacement method. Star-shaped POSS-PCL-PU films possessed a higher porosity of $75.7 \pm 7.4 \%$ compared with the porosity of the linear PCL-PU films of $43.1 \pm 4.4 \%$. The significantly higher porosity with greater interconnectivity of pores enables more efficient transport of nutrients in the scaffolds without compromising the mechanical stability. Their hydrophobicity was further assessed to verify the wettability of the films by using a contact angle goniometer. The star-shaped POSS-PCL-PU films were found to be slightly hydrophilic, with a contact angle of $89^{\circ}$, while the linear PCL-PU films were found to be hydrophobic, with a contact angle of $98^{\circ}$. The POSS moieties have weak polarity, because of its enclosed cubic silica structure, and are distinctly hydrophobic. ${ }^{8,25}$ However, this hydrophobic nature of POSS was not observed in the star POSS-PCL-PU films. The reason for the slight hydrophilicity observed in the star-shaped POSS-PCL-PU films could be because of their higher porosity, thereby causing more water to be adsorbed at the surface and resulting in a decrease in the wettability of films, which further affects the cell adhesion. A similar finding was observed earlier in POSS-polycarbonate urethane foam matrices, ${ }^{16}$ and the increased hydrophilicity with higher POSS content was attributed to the increased porosity and pore size.

To evaluate the feasibility of using star-shaped POSS-PCL-PU as a promising scaffold material for tissue engineering, it is important to study their degradation profiles to determine the suitable bioapplications. Hence, degradation studies were performed on star-shaped POSS-PCL-PU and linear PCL-PU films over 52 weeks. Figure 3 shows the weight loss profiles of the two films in PBS buffer at $37^{\circ} \mathrm{C}$. For the POSS-PCL-PU films, although there was a negligible weight loss of $<1 \%$ in the initial 24 weeks of degradation, substantial weight loss of 9\% was observed at 36 weeks, and a further 9\% weight loss was observed at the end of 52 weeks. The slow initial rate of hydrolytic degradation can be explained by the hydrophobic nature and the shielding effect of the POSS moieties. ${ }^{26}$ The rate of degradation increased by many fold only after the POSS moieties were broken down. The degradation rate can be determined by varying POSS content; a higher POSS content corresponds to a slower degradation rates. ${ }^{27}$ Together with the mechanical integrity, the degradation rate of the POSS-PCL-PU films can be controlled to achieve a scaffold that degrades slowly during the initial period, followed by rapid degradation at the later phase after the growth of the cells. This behavior is desired because the scaffold's structural integrity is required in the initial phase for cells to attach and proliferate. At the later phase, as the cells have grown to form tissues, the scaffold has fulfilled its intended function and should be quickly eliminated from body. In comparison, linear PCL-PU films exhibited a gradual weight loss over 52 weeks. The weight loss profile of the PCL-PU films was observed to be linear and consistent, while that of POSS-PCL-PU films was found to be biphasic because of the protective effect of the POSS cages during the initial phase. The overall weight loss of the PCL-PU films at the end of 52 weeks was low, at a $\sim 11 \%$, which is because of the hydrophobicity and the semi-crystalline structure of the PCL backbone, resulting in slow degradation. ${ }^{28}$ However, PCL-PU copolymers were reported to
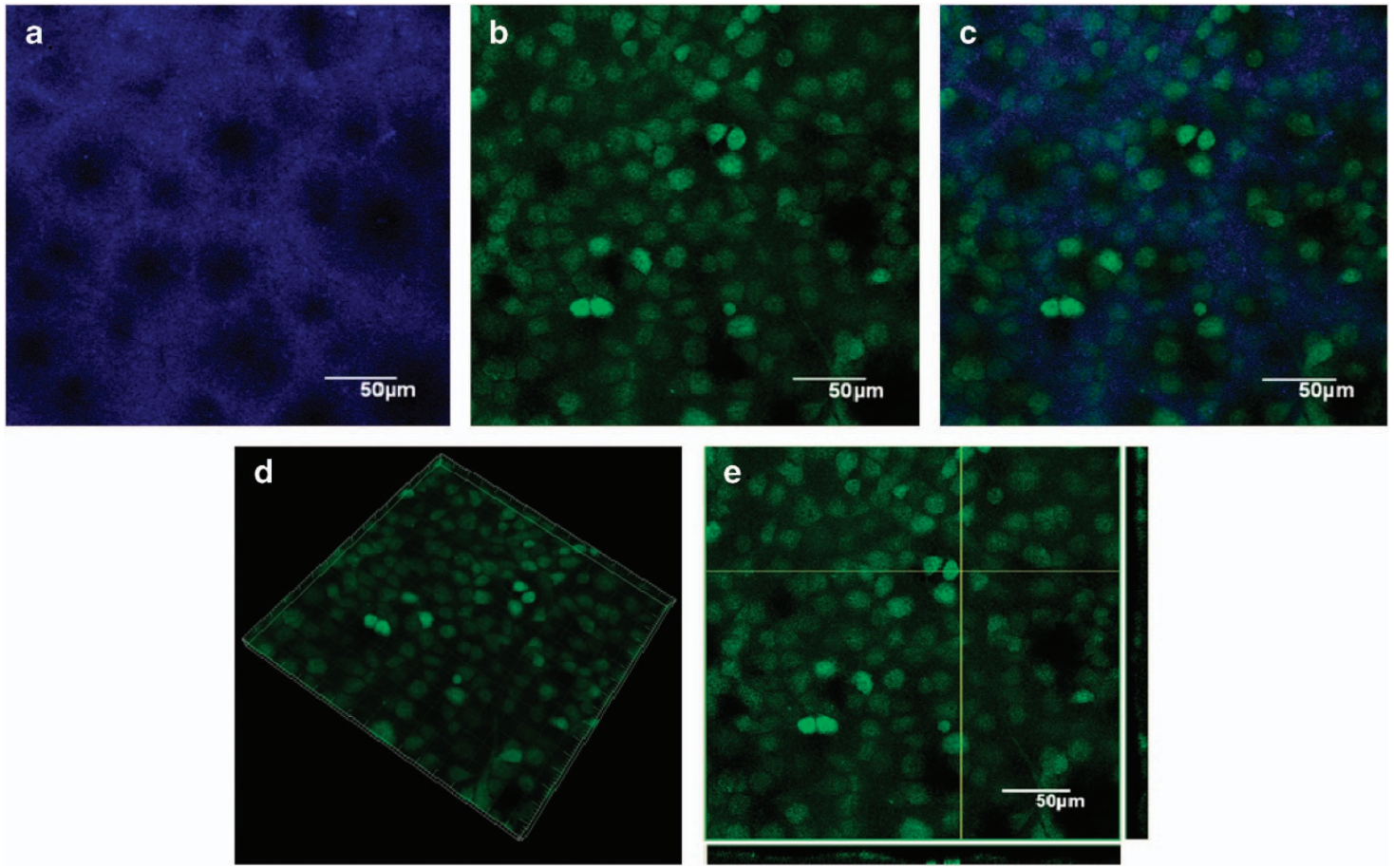

Figure 7 Confocal images of fluorescein diacetate-stained CCD-112 CoN cells after 10-day growth on star-shaped POSS-PCL-PU film. (a) Auto-fluorescing image of characteristic spherulites in film under $405 \mathrm{~nm}$ excitation (blue channel). (b) Projected image of fluorescein diacetate-stained cells in $z$ direction under $488 \mathrm{~nm}$ excitation (green channel). (c) Overlay image of these two channels. The fibroblast cells were observed to form a confluent layer on the surface features of the film. Imaris images showing (d) three-dimensional projection and (e) cross-section view of cell growth in $650 \mu \mathrm{m} \times 650 \mu \mathrm{m}$ area of the film. Scale $\operatorname{bar}=50 \mu \mathrm{m}$. 
be stiff and hydrophobic, causing compatibility issues with soft tissues, thereby limiting their use in tissue engineering applications. ${ }^{29}$

The morphology of the degraded films was also monitored using an SEM during the in vitro degradation for 52 weeks (Figure 4). The morphological changes of the star-shaped POSS-PCL-PU and the linear PCL-PU films were found to be minimal in the first 24 weeks.
For the star-shaped POSS-PCL-PU film (Figures 4a and e), the surface of the spherulites became less smooth, and the gaps between spherulites disappeared at 4 weeks of degradation. After 4 weeks, more tiny particles appeared on the surface with increased time. For the linear PCL-PU film (Figures $4 \mathrm{f}$ and $\mathrm{j}$ ), the surface of spherulites still remained smooth in the first 4 weeks, and then cracks were
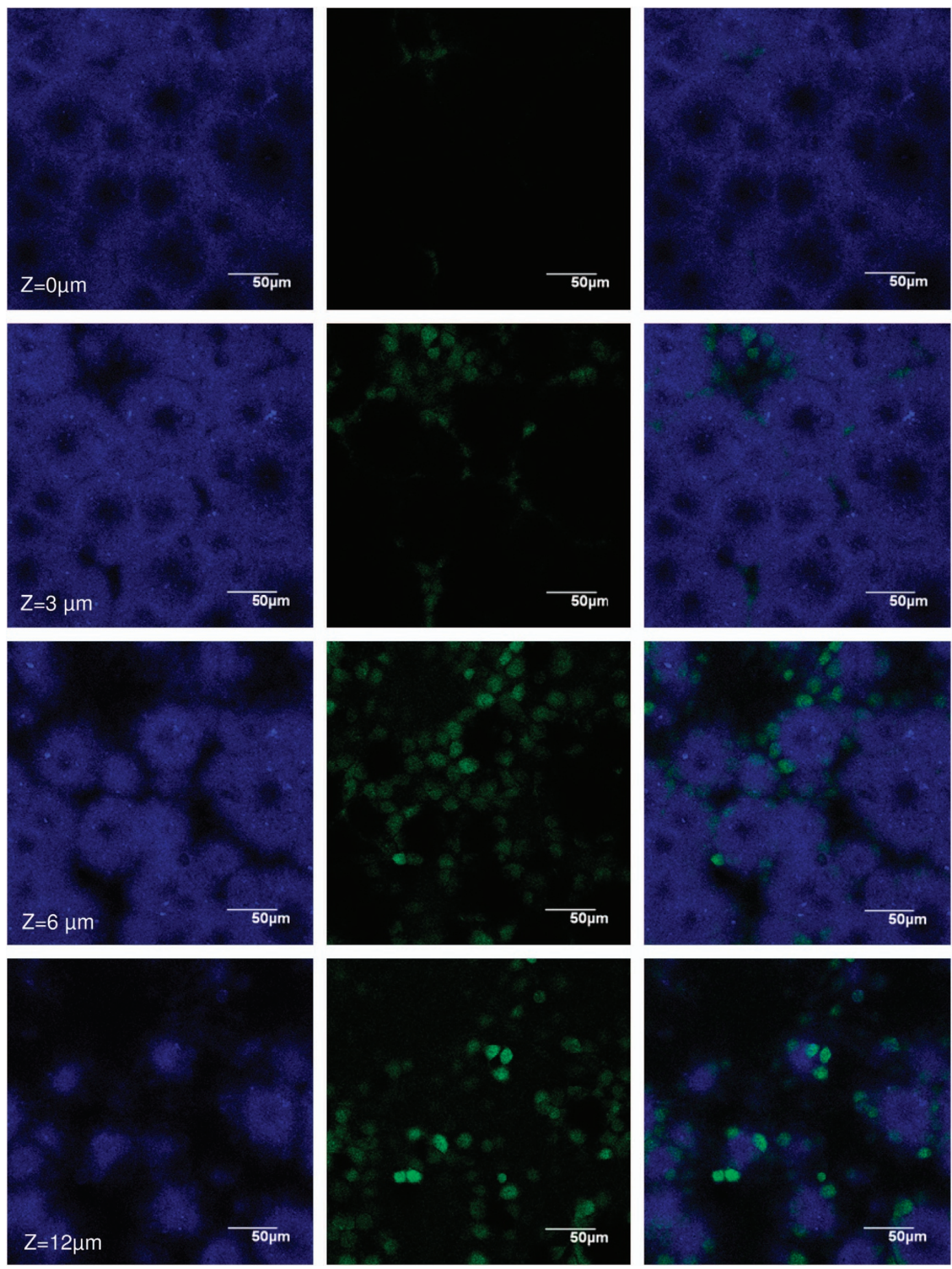

Figure 8 Confocal $z$-sectioning images of fibroblasts growth around spherulites of star-shaped POSS-PCL-PU film at $z=0,3,6$ and $12 \mu \mathrm{m}$, shown in blue, green and combined channels. Scale bar $=50 \mu \mathrm{m}$. 
formed on the surface after week 24. At higher magnifications, smaller cracks were observed in the POSS-PCL-PU film (Figures $5 \mathrm{a}$ and $\mathrm{b}$ ) and the PCL-PU film (Figures $5 \mathrm{c}$ and $\mathrm{d}$ ) after the 24 weeks of degradation. The porous regions were found to become separate fibril strands in the PCL-PU film (Figure 5d). At the end of 52 weeks, the distinct shape of spherulites was no longer observed, whereas more and larger cracks were observed on the partially degraded film. During the later phase of the study, both films became more brittle and broke into tiny fragments easily during handling because of the reduced molecular weight of polymeric films after degradation.

After incubating $24 \mathrm{mg}$ of POSS-PCL-PU or PCL-PU films in $2 \mathrm{ml}$ of PBS at $37^{\circ} \mathrm{C}$ for 8 weeks, the degraded products from the films were extracted into PBS and used for cytotoxicity analysis via alamarBlue (Life Technologies) reagent. The obtained solutions (12 $\left.\mathrm{mg} \mathrm{ml}^{-1}\right)$ were further diluted into different concentrations $(0.75,1.5$, 3.0 and $6.0 \mathrm{mg} \mathrm{ml}^{-1}$ ) and then incubated with fibroblast cells (CCD$-112 \mathrm{CoN}$ ) at a density of $2.0 \times 10^{4}$ cells $\mathrm{cm}^{-2}$ in 96 -well plates. The cell viability remained high at $>95 \%$ after $48 \mathrm{~h}$, demonstrating the negligible cytotoxicity of the side products of both films (Figure 6). Note that increasing concentrations of degradation products did not have any adverse impact on cell proliferation, and thus, the film is safe for use in biological applications.

Biocompatibility of the POSS-PCL-PU films was investigated by seeding CCD-112 CoN cells onto them and then incubating for 10 days before assessing the cell attachment and growth using confocal microscopy. After staining with fluorescein diacetate, the live fibroblast cells appeared as a green confluent monolayer on the slightly hydrophilic star-shaped POSS-PCL-PU film (Figures $7 \mathrm{a}$ and c). Optical sectioning along the $z$ axis illustrates the growth at different focal planes and demonstrated that the fluorescein diacetate-stained green fibroblast cells were able to adhere and proliferate around and on the distinct spherulites features of the POSS-PCL-PU films (Figure 8 and Supplementary Figure S2). Imaris imaging software was used to further render three-dimensional visualization and crosssection views of the fibroblasts in the green channel (Figures $7 \mathrm{~d}$ and e). The $x z$ and $y z$ view of the cross-sectional image demonstrated the growth of cells at different layers along the $z$ axis. Another cell line, Mb231-GFP, was also observed to form a confluent layer on the starshaped POSS-PCL-PU film (Supplementary Figure S3). However, it is the presence of POSS in PCL-PU that improves the cell-substrate affinity because the moderate hydrophilicity provides a good support for the cell adhesion, ${ }^{30}$ and the intrinsic roughness at sub-micrometer/ nanometer scales enhances the contact guidance for cell interactions and growth. ${ }^{31}$ All these favorable factors (excellent biocompatibility, high porosity, moderate hydrophilicity and rough surface nanotopography) present in the star-shaped POSS-PCL-PU film created a very conducive environment for enhanced cell interactions, adhesion and proliferation. Future experiments will be focused on improving the bioactivity of the film for active interaction with the cellular components of the engineered tissues to facilitate and regulate their activities by including biological cues, such as cell-adhesive ligands, to enhance attachment or physical cues, such as topography, to influence cell morphology and alignment.

\section{CONCLUSIONS}

Star-shaped POSS-PCL-PU was synthesized and used to prepare highly porous films that exhibited controlled and slow initial degradation as biodegradable scaffold biomaterials for tissue engineering applications. For such applications, another important feature is the ability to control the degradation rate of the films to maintain their mechanical stability until the intended function is fulfilled before degrading into non-toxic byproducts. In addition, the film and its metabolic products exhibited excellent biocompatibility, with no adverse effects and hindrance on cell growth upon incubation with cells for $48 \mathrm{~h}$. The inclusion of POSS in PCL-PU strengthens the cellsubstrate affinity of the film by improving its hydrophilicity to provide a greater support for the cell adhesion and by increasing its intrinsic roughness at the sub-micrometer/nanometer level to enhance contact guidance for cell interactions and growth. The combination of organic and inorganic functionalities in the unique nanocomposite POSSPCL-PU offers superior properties compared to traditional PCL-PU. In summary, the star-shaped POSS-PCL-PU film produced in this study, with its unique nanotopography, exhibits excellent biocompatibility, high porosity and controlled degradability, which favor cell adhesion and proliferation, thereby making it a great candidate as a scaffold biomaterial for use in tissue engineering.

\section{CONFLICT OF INTEREST}

The authors declare no conflict of interest.

\section{ACKNOWLEDGEMENTS}

This study was supported by the Institute of Materials Research and Engineering (IMRE), the Agency of Science Technology and Research $\left(A^{*}\right.$ STAR $)$ in Singapore and the National University of Singapore. We thank Dr Yap Fung Ling from IMRE for her help with performing the AFM measurements and express our thanks to the Confocal Microscopy Unit, National University Health System.

1 Chan, B. P. \& Leong, K. W. Scaffolding in tissue engineering: general approaches and tissue-specific considerations. Eur. Spine J. 17, 467-479 (2008).

2 Yang, S., Leong, K., Du, Z. \& Chua, C. The design of scaffolds for use in tissue engineering. Part I. Traditional factors. Tissue Eng. 7, 679-689 (2001).

3 Chen, G., Ushida, T. \& Tateishi, T. Scaffold design for tissue engineering. Macromol. Biosci. 2, 67-77 (2002).

4 Le, X., Poinern, G. E. J., Ali, N., Cassandra, M. B. \& Fawcett, D. Engineering a biocompatible scaffold with either micrometre or nanometre scale surface topography for promoting protein adsorption and cellular response. Int. J. Biomater. 2013, PMC3600176 (2013).

5 Shoichet, M. S. Polymer scaffolds for biomaterials applications. Macromolecules $\mathbf{4 3}$ 581-591 (2010)

6 She, M.-S., Lo, T. Y., Hsueh, H.-Y. \& Ho, R.-M. Nanostructured thin films of degradable block copolymers and their applications. NPG Asia Mater 5, e42 (2013).

7 Liu, Y., Yang, X., Zhang, W. \& Zheng, S. Star-shaped poly( $\varepsilon$-caprolactone) with polyhedral oligomeric silsesquioxane core. Polymer 47, 6814-6825 (2006).

8 Lai, Y. S., Tsai, C. W., Yang, H. W., Wang, G. P. \& Wu, K. H. Structural and electrochemical properties of polyurethanes/polyhedral oligomeric silsesquioxanes (PU/ POSS) hybrid coatings on aluminum alloys. Mater. Chem. Phys. 117, 91-98 (2009).

9 Raftopoulos, K. N., Pandis, C., Apekis, L., Pissis, P., Janowski, B., Pielichowski, K. \& Jaczewska, J. Polyurethane-POSS hybrids: molecular dynamics studies. Polymer 51, 709-718 (2010).

10 Matějka, L., Murias, P. \& Pletil, J. Effect of POSS on thermomechanical properties of epoxy-POSS nanocomposites. Eur. Polym. J. 48, 260-274 (2012).

11 Tanaka, K. \& Chujo, Y. Chemicals-inspired biomaterials: developing biomaterials inspired by material science based on POSS. Bull. Chem. Soc. Jpn. 86, 1231-1239 (2013).

12 Mya, K. Y., Gose, H. B., Pretsch, T., Bothe, M. \& He, C. Star-shaped POSSpolycaprolactone polyurethanes and their shape memory performance. J. Mater. Chem. 21, 4827-4836 (2011).

13 Lee, K. S. \& Chang, Y. W. Thermal and mechanical properties of poly(e-caprolactone)/ polyhedral oligomeric silsesquioxane nanocomposites. Polym. Int. 62, 64-70 (2013).

14 Nanda, A. K., Wicks, D. A., Madbouly, S. A. \& Otaigbe, J. U. Nanostructured polyurethane/POSS hybrid aqueous dispersions prepared by homogeneous solution polymerization. Macromolecule 39, 7037-7043 (2006).

15 Raghunath, J., Georgiou, G., Armitage, D., Nazhat, S. N., Sales, K. M., Butler, P. E. \& Seifalian, A. M. Degradation studies on biodegradable nanocomposite based on polycaprolactone/polycarbonate $(80: 20 \%)$ polyhedral oligomeric silsesquioxane. J. Biomed. Mater Res 91A, 834-844 (2009).

16 Oseni, A. O., Butler, P. E. \& Seifalian, A. M. The application of POSS nanostructures in cartilage tissue engineering: the chondrocyte response to nanoscale geometry. J. Tissue Eng. Regen. Med. (e-pub ahead of print 10 April 2013; doi:10.1002/term.1693).

$17 \mathrm{Wu}$, J. \& Mather, P. T. POSS polymers: physical properties and biomaterials applications. J. Macromol. Sci Polym. Rev. 49, 25-63 (2009). 
18 Knight, P. T., Kirk, J. T., Anderson, J. M. \& Mather, P. T. In vivo kinetic degradation analysis and biocompatibility of aliphatic polyester polyurethanes. J Biomed. Mater. Res. 94A, 333-343 (2010).

19 Neumann, D., Fisher, M., Tran, L. \& Matisons, J. G. Synthesis and characterization of an isocyanate functionalized polyhedral oligosilsesquioxane and the subsequent formation of an organic-inorganic hybrid polyurethane. J. Am. Chem. Soc. 124, 13998-13999 (2002)

20 Liu, H. \& Zheng, S. Polyurethane networks nanoreinforced by polyhedral oligomeric silsesquioxane. Macromol. Rap. Commun. 26, 196-200 (2005).

21 Mya, K. Y., Wang, Y., Shen, L., Xu, J., Wu, Y., Lu, X. \& He, C. Star-like polyurethane hybrids with functional cubic silsesquioxanes: preparation, morphology, and thermomechanical properties. J. Polym. Sci. A Polym. Chem. 47, 4602-4616 (2009).

22 Yeh, C. C., Li, Y. T., Chiang, P. H., Huang, C. H., Wang, Y. \& Chang, H. I. Characterizing microporous PCL matrices for application of tissue engineering. J. Med. Biol. Eng. 29 92-97 (2009).

23 Yang, J., Shi, G., Bei, J., Wang, S., Cao, Y., Shang, Q., Yang, G. \& Wang, W. Fabrication and surface modification of macroporous poly(L-lactic Acid) and poly(L-lactic-Coglycolic acid) (70/30) cell scaffolds for human skin fibroblast cell culture. J. Biomed. Mater. Res. $-A$ 62, 438-446 (2002).

24 Chua, K. N., Lim, W. S., Zhang, P., Lu, H., Wen, J., Ramakrishna, S., Leong, K. W. \& Mao, H. Q. Stable immobilization of rat hepatocyte spheroids on galactosylated nanofiber scaffold. Biomaterials 26, 2537-2547 (2005).

25 Tanaka, K. \& Chujo, Y. Unique properties of amphiphilic POSS and their applications. Polym. J. 45, 247-254 (2013).

26 Kannana, R. Y., Salacinskia, H. J., Odlyhab, M., Butlerc, P. E. \& Seifaliana, A. M. The degradative resistance of polyhedral oligomeric silsesquioxane nanocore integrated polyurethanes: an in vitro study. Biomaterials 27, 1971-1979 (2006).
27 Knight, P. T., Lee, K. M., Chung, T. \& Mather, P. T. PLGA-POSS end-linked networks with tailored degradation and shape memory behavior. Macromolecules 42 6596-6605 (2009).

28 da Silva, G. R., da Silva-Cunha, A. J., Behar-Cohen, F., Ayres, E. \& Oréfice, R. L. Biodegradation of polyurethanes and nanocomposites to non-cytotoxic degradation products. Polym. Degrad. Stabil. 95, 491-499 (2010).

$29 \mathrm{Gu}$, X., Wu, J. \& Mather, P. T. Polyhedral oligomeric silsesquioxane (POSS) suppresses enzymatic degradation of PCL-based polyurethanes. Biomacromolecules 12 3066-3077 (2011).

30 Wang, L., Sun, B., Ziemer, K. S., Barabino, G. A. \& Carrier, R. L. Chemical and physical modifications to poly(dimethylsiloxane) surfaces affect adhesion of caco-2 cells. J. Biomed. Mater. Res. A 93, 1260-1271 (2010).

31 Saltzman, W. M. \& Kyriakides, T. R in Principles of Tissue Engineering 3rd Edn eds Lanza R. P., Langer R., Vacanti J. Ch. 20 279-296 Elsevier, Academic Press: San Diego, USA (2011).

(c) (i)(2) (2) This work is licensed under a Creative Commons Attribution-NonCommercial-ShareAlike 4.0 International License. The images or other third party material in this article are included in the article's Creative Commons license, unless indicated otherwise in the credit line; if the material is not included under the Creative Commons license, users will need to obtain permission from the license holder to reproduce the material. To view a copy of this license, visit http://creativecommons.org/licenses/bync-sa/4.0/

Supplementary Information accompanies the paper on the NPG Asia Materials website (http://www.nature.com/am) 